\title{
TOWARDS A BETTER UNDERSTANDING OF SCREEN PRINT THICKNESS CONTROL
}

\author{
R. J. HORWOOD \\ Electronic Technology Department, British Aircraft Corporation Ltd., Guided Weapons Division, \\ GPO Box 77, Filton House, Bristol BS99 7AR, U.K.
}

(Received June 6, 1974; in final form July 20, 1974)

\begin{abstract}
In recent years, a more scientific approach to the age-old craft of silk screen printing has resulted in the evolution of complex precision-built printing machines for use in the electronics micro-circuit industry. Even so, our knowledge of the physical processes involved in screen printing is still far from complete. An attempt is made here to provide a better understanding of the screen printing mechanism and more specifically of print thickness control. Two different printing modes are described and the effect of and interaction between some of the more important machine parameters in this respect are discussed. A simple pillar theory is offered which allows a prediction to be made of the quantity of ink fundamentally deposited by a given screen.
\end{abstract}

\section{INTRODUCTION}

The screen printing process, known for many hundreds of years, enables high quality patterns to be repetitively produced at high rates and low cost, In view of these attributes it is not surprising to find such a process has been adopted by the electronics industry for the deposition of thick film passive components, conductors, capacitors and resistors on to insulating substrates as the basis of a complex hybrid microcircuit manufacturing operation. Whereas high definition printing with this process has always been of prime importance, now, especially with new inks, of equal moment is the need to control print thickness precisely, particularly with resistive elements if target resistance values are to be approached.

More than anything else it is this new requirement which, in a single decade, has caused such activity that the screen printing process has emerged from the status of a craft, little understood, to a process operation the control of which has now some scientific basis.

The controlled printing of resistors has proved to be a particularly difficult area; the problem is to deposit precise amounts of resistive ink repeatedly, which on subsequent high temperature processing materialize into precise valued resistor elements.

Much has been written ${ }^{1,2,3}$ regarding the essential features of screen printing control and not all authors have agreed on the relative importance of the very many parameters involved. This perhaps is due to a failure to recognise the interaction some parameters have on others.

It is our purpose here, concentrating on print thickness control, to examine in turn the various facets of screen printing - the ink, the screen, the major printing parameters and the interaction of some of these to provide a better understanding of the printing mechanism.

\section{SCREEN PRINTING CONCEPT}

A basic concept of screen printing is that ink is transferred, in a controlled manner, through the apertures of a mesh and deposited on to a substrate. This process is accomplished by a flexible squeegee stroking the ink across the screen surface. In so doing the screen is usually depressed into line contact with the substrate effectively sealing off a line of mesh apertures and filling the cells so formed with ink. The mesh-substrate line of contact advances with the squeegee stroke allowing the mesh to peel away from the substrate behind the squeegee and depositing its ink charge on to the substrate in the manner depicted in Figure 1.

Any required pattern can then be printed by blocking off appropriate mesh apertures with a photo emulsion layer to form a printing screen, the pattern definition being obviously dependent on the mesh count (number of threads per inch). 


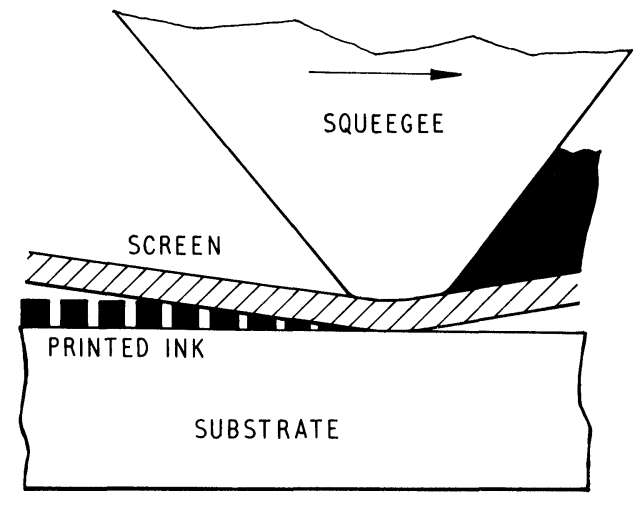

FIGURE 1 Screen printing concept.

Both resistive and conductive printing inks comprise metal or metallic oxide particles and a glass frit held together with a suitable binder, and having a volatile organic vehicle added to provide the type of ink flow characteristics required for screen printing.

Ideally, the ink viscosity should be low enough for easy flow through the mesh under the shearing stress encountered during the screening, but high enough when deposited, and with the shearing stress removed, to prevent lateral flow. Fluids exhibiting this kind of behaviour - when at rest they are extremely viscous but flow quite readily under moderate shearing stress - are termed pseudo-plastic.

During the subsequent heated drying stage it is essential, if additional pattern flow is to be prevented, that the organic vehicle evaporates quickly to compensate for the lowering of ink viscosity which occurs with rising temperature.

The residual dry print is a homogeneous mixture loosely attached to the substrate, and having approximately half the wet print thickness. The principal factor governing the reduction in thickness on drying is the solid-to-liquid ratio of the wet print constituents.

The drying process is followed by a firing cycle having a precise temperature-time relationship for periods usually in excess of 45 minutes, with peak temperatures commonly between $750^{\circ} \mathrm{C}$ and $950^{\circ} \mathrm{C}$. In this process the binder is first burnt off followed by melting of the glass frit. The molten glass wets the substrate, and in the case of resistive inks provides a matrix wherein the metallic and/or metallic oxide components, formed at the higher temperatures, are held to provide a continuous electrical path.

The resistivity of the finished element is strongly dependent on the glass-metal ratio, there being a range of specific values offered by all ink manufacturers.

In the case of conductive inks the glass content mainly settles at the conductor-substrate interface, providing the necessary adhesion for the predominantly metallic conduction layer.

Since the length and width dimensions of a thick film resistor are fixed by the initial screen pattern, the resistance value is determined by the fired print thickness which usually is in the order of 10-15 microns. Assuming a simple inverse relationship between resistance and thickness, even a perfectly defined print requires this thickness control to better than 1.5 microns to achieve a $10 \%$ tolerance resistor. When it is further considered that the ends of the resistor normally rise up to overlap the conductor terminations, it is little wonder that print thickness control has proved to be so difficult.

A normal production target of resistance-value after firing is often $-40+0 \%$, so that for these reasons one has to accept that for close tolerance resistors additional post firing adjustment (trimming) is essential (whereby removal of resistor material increases the resistance value to a higher and more precise value). Dimensional allowances for such adjustment must be made in the pattern design. This does not make print thickness control any less desirable, since the more accurate the as-fired resistor value, the less production time is required for its final adjustment to value.

A secondary point is that if adjustment of the resistor value is made by the commonly used method of cutting away resistive material, power dissipation per unit area is increased in the remaining region. To ensure that the dissipation per unit area is not excessive, the dimensions of the resistor need to be increased. The larger the degree of trimming to be catered for, the more substrate area is consumed, and the lower is the component packing density.

The amount of ink required to be deposited to produce desired resistance values can be gauged by empirical means only and once determined it may require to be adjusted from time to time. Such adjustments may be needed to compensate for volatile losses from the ink during normal usage. Evaporation of the organic vehicle after prolonged atmospheric exposure will result in an increase in the solid-to-liquid ratio and a readily noticeable increase in ink viscosity. Unless compensated for by adjusting printing conditions or restoring the ink by the addition of thinners, thicker dry prints and hence lower resistance values will result. From all this it can be judged that only fair correlation can be expected 
of wet or dry print thickness with final as-fired resistance value. Nevertheless the ink manufacturer often indicates a dry print thickness to be aimed for and it is possible to choose on theoretical grounds a suitable mesh type and emulsion combination which will achieve approximately that value.

\section{THE SCREEN}

The screen comprises a tensioned mesh material, having appropriate apertures blocked to form the desired pattern, mounted on a rigid frame. Mesh material, once the prerogative of silk, is nowadays woven from stainless steel as well as a variety of man-made fibres.

The stencil pattern is photographically produced on a sensitized emulsion adhered to the mesh. The emulsion thickness as well as that of the mesh plays an important part in determining the thickness of the ink prints.

A screen of uniformly woven mesh in silk or synthetic fibre will appear as in Figure 2 where $d$ is the thread diameter and $T$ the mesh count giving a mesh thickness of $2 d$. On the other hand commercially available stainless steel mesh will appear more like Figure 3. Here in weaving, because of the greater

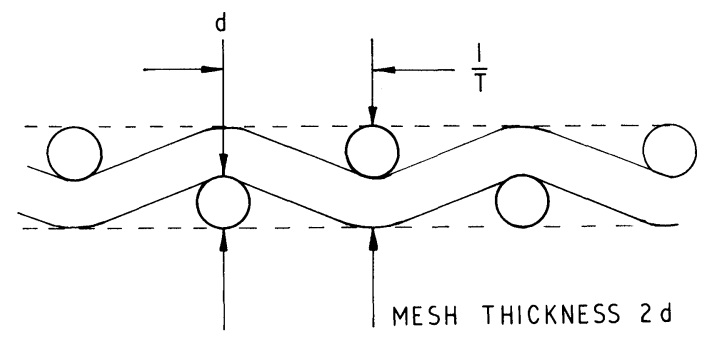

FIGURE 2 Section of silk mesh.

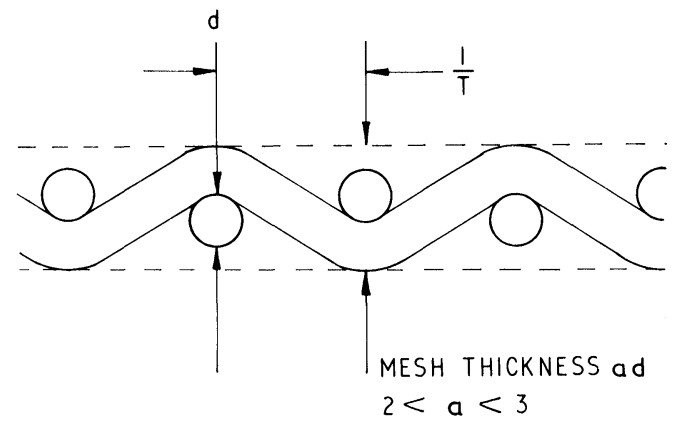

FIGURE 3 Section of stainless steel mesh. rigidity of stainless steel threads, the warp lines have larger amplitude than the weft lines resulting in an overall mesh thickness $a d(2<a<3)$. Being also a function of weaving tensions, $a$ varies from manufacturer to manufacturer. It usually lies between 2.3 and 2.6 and has a significance in printing thickness control which is often overlooked.

Just how mesh parameters affect screened print thickness is indicated in the following pillar theory.

\section{THE PILLAR THEORY}

The ink is initially deposited as columns or pillars conforming to the mesh cell dimensions and spacings sensibly having a height equal to the total screen thickness, that is the combined mesh and emulsion thickness $(a d+e)$; see Figure 4. For such a mesh of $T$ threads per inch (tpi) and wire diameter $d$ each pillar of wet ink has a volume

$$
\left(\frac{1}{T}-d\right)^{2}(a d+e)
$$

Since there are $T^{2}$ such pillars in a unit square of area the total ink volume per unit area is

$$
v=\left(\frac{1}{T}-d\right)^{2}(a d+e) T^{2}
$$
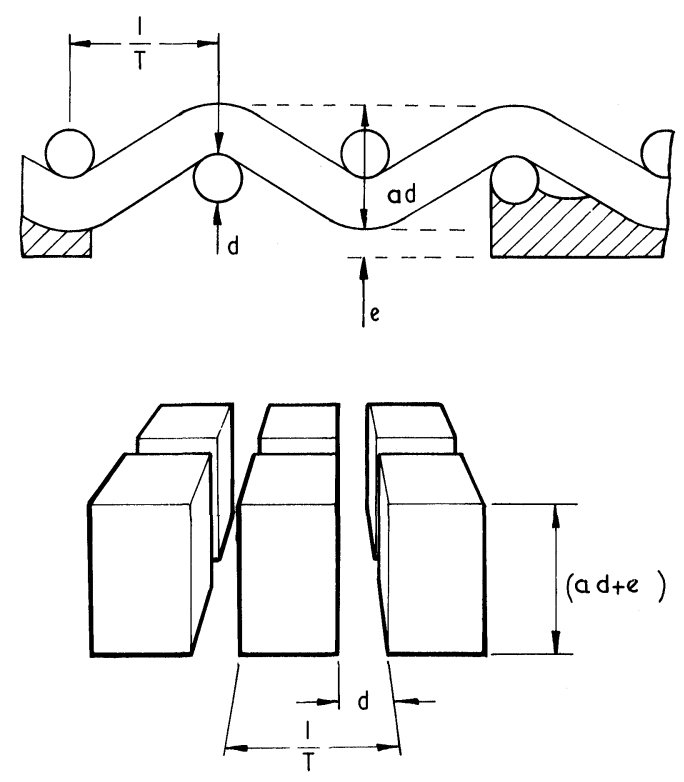

FIGURE 4 Deposited ink pillars. 
Once deposited, some lateral pillar flow is ensured by residual gravitational stress. If the pillar spacing is small enough merging occurs aided by surface tension resulting in a continuous print thickness $t$. Volatile losses are small enough to neglect during this levelling period and therefore

$$
t=v=\left(\frac{1}{T}-d\right)^{2}(a d+e) T^{2}
$$

Consider thus a printing screen comprising a stainless steel mesh of 200 tpi with standard wire diameters of 0.0016 in., having a mesh thickness $2.5 d$ and an emulsion thickness of 0.001 in. Prints from such a screen will yield a wet thickness of

$$
t=\left(\frac{1}{200}-0.0016\right)^{2}(2.5 \times 0.0016+0.001)(200)^{2}
$$

or $t=(0.0034 \times 200)^{2}(0.005)=0.00231 \mathrm{in}$.

The same screen made with the equivalent nylon mesh where the mesh thickness is only $2 d$ would yield a wet print thickness of

$$
\begin{aligned}
t & =(0.0034 \times 200)^{2}(0.0042) \\
& =0.00194(16 \text { percent less })
\end{aligned}
$$

The dried print thickness very much depends on the solid/liquid ratio of the ink and usually approximates to 50 percent of the wet print thickness; for any specific ink an accurate figure may be obtained empirically.

The usefulness of this theory is demonstrated by the following actual example.

A specific resistor ink, nominally of $100 \mathrm{k} \Omega$ per square resistivity, when printed through a 165 tpi stainless steel screen at slow speed resulted in prints having, when dried, a thickness of 0.00135 in. which on firing achieved only $60 \mathrm{~K} \Omega$ per square resistivity.

The prints here were obviously too thick and it was desired to know what improvement would result from the use of a 200 tpi stainless steel screen.

The pillar theory predicts a wet print thickness of 0.0022 in. for any ink printed slowly through the 165 tpi screen having wire diameters of $0.002 \mathrm{in}$. and $a=2.5$, there being negligible emulsion thickness.

For the ink in question this means a dry-to-wet print thickness ratio of $0.00135 / 0.0022$ or 0.61 .

The only 200 tpi screen available had wire diameters of 0.0016 in. and $a=2.3$, again with negligible emulsion thickness. The pillar theory predicts a wet print thickness from such a screen of 0.0017 in. which for the particular ink sample in question will reduce to $0.0017 \times 0.61=0.001 \mathrm{in}$. when dried, and by simple inverse relation will result in a sheet resistivity when fired of $81 \mathrm{~K} \Omega$ per square.

Actual measurements of dry print thickness averaged $0.00095 \mathrm{in}$. and mean sheet resistivity when fired was $85 \mathrm{~K} \Omega$ per square for samples prepared with this screen.

Had a similar 200 tpi nylon screen been available with $a=2$, the pillar theory predicts a sheet resistivity of $90 \mathrm{~K} \Omega$ per square.

Print thickness predictions of this nature can be confidently expected to be accurate within 10 percent provided always that printing conditions are strictly maintained constant.

It can be thus seen that within certain limits, any ink print thickness can be achieved by the proper choice of screen dimensions. Usually, however, the screen is regarded as a coarse print thickness control, the fine control being left to the adjustment of one or more parameters of the screen printing machine.

To enable one to picture what effect the various printer parameters have on print thickness the following hypothesis on the screen printing mechanism is presented.

\section{A HYPOTHESIS OF THE SCREEN PRINTING MECHANISM}

Figure 5(i) shows a tensioned screen $M$ set parallel to and spaced at distance $D$ from a substrate. In Figure 5(ii) the wedge shaped elastic squeegee under the action of a force $F$ depresses the screen $M$ to a level $L$ (limited by an adjustable stop $S$ ) slightly lower than that of the substrate surface. In this static condition the vertical component of the screen tensions $T$ are balanced by the elastic compression reaction of the now deformed squeegee tip.

Figure 5(iii) shows printing in progress. The squeegee, traversing the screen with velocity $V$, presses it into line contact with the substrate under the full force $F$ (being now free of the stop $S$ ). Ink under hydraulic pressure, resulting from the squeegee movement and angled tip, is forced through the screen apertures to be deposited on to the underlying substrate.

The reaction $R$ of this hydraulic pressure on the squeegee has a vertical component in opposition to the force $F$. The greater the stroke speed $V$ the greater will be the reaction $R$, whilst the vertical component of $R$ will be further enhanced by the decrease in the contact angle $\theta$ as the elastic squeegee tip further deforms under the action of the horizontal 


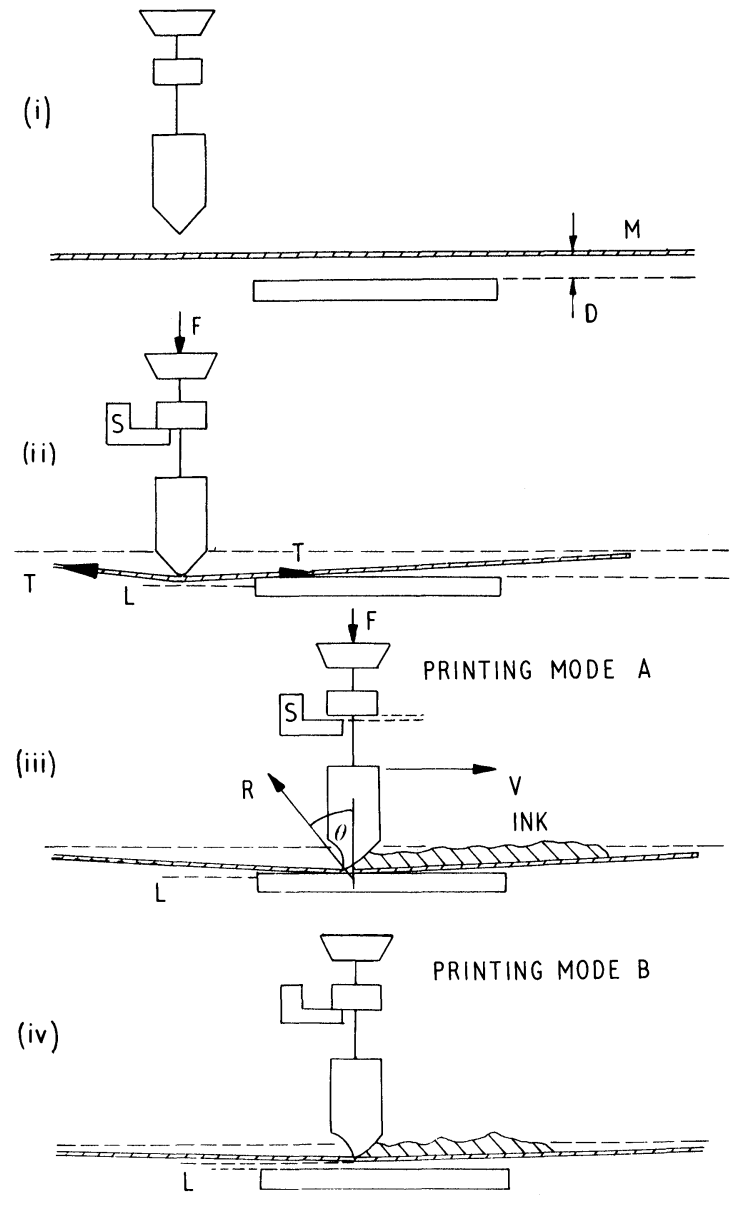

FIGURE 5 The screen printing mechanism.

component of $R$. The overall effect is a tendency for the squeegee to be slightly lifted off the screen, resulting in increased print thicknesses.

A similar situation prevails when the pre-set stop position is adjusted for the level $L$ to lie slightly above the substrate surface (see Figure 5(iv)) and the screen is not pressed into intimate line contact with the substrate. Again thicker prints will result.

So for printing mode A (Figure 5(iii)) variation of print thickness is achieved by adjusting the squeegee traverse speed, whilst in printing mode B (Figure 5(iv)) print thickness is controlled by the pre-set adjustment of level $L$ by the stop position $S$, all other controls being maintained constant. Satisfactory prints can be achieved in this latter mode even when the level $L$ is as much as $0.005 \mathrm{in}$. or more above the substrate surface and this method has the virtue that its prints are unaffected by previously deposited thick film layers on the substrate.
Unfortunately, since in this mode the squeegee remains at constant level during its traverse, normal variations encountered in substrate thickness will influence the print thicknesses.

The squeegee level in mode A follows the substrate surface and it therefore accommodates normal substrate thickness variations extremely well. Previously deposited layers can, however, slightly influence the printed thickness.

\section{DEPENDENCE OF PRINT THICKNESS ON SQUEEGEE SPEED}

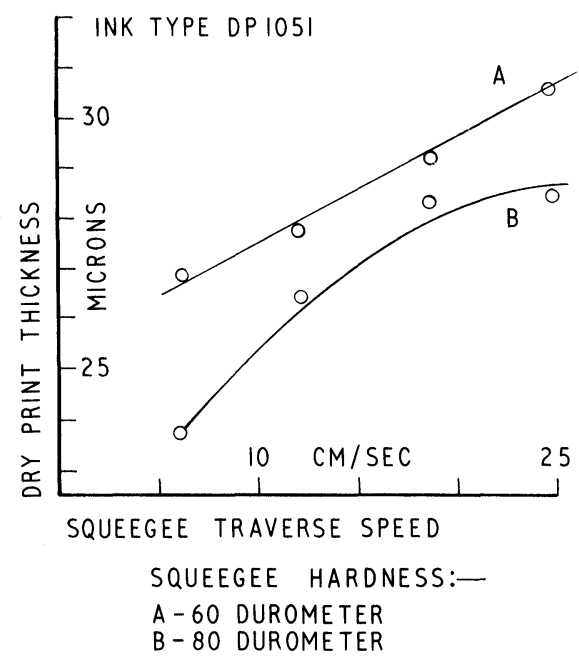

FIGURE 6 Dependence of print thickness on squeegee speed.

Figure 6 displays the typical dependence of dry print thickness on squeegee traverse velocity for two similarly shaped squeegees of differing hardness, all other printer parameters being maintained sensibly constant. Dry print thickness measurements, never easy to make, were assessed from Talysurf traces.

Since the hydraulic pressure required to cause ink flow through the screen at a given rate is closely related to the ink rheology, it is to be expected that some inks are more sensitive to printing stroke speed, in respect of print thickness, than others. This is demonstrated in Figure 7 where the mean resistivities (more accurately measured than print thickness) of five inks in the BIROX 1000 series are plotted against squeegee stroke speed. Separate plots are shown for two different squeegee materials, all other printing parameters being kept constant. The mean sheet resistivities are determined for resistor samples all of the same size, $5 \mathrm{~mm} \times 1 \mathrm{~mm}$. 


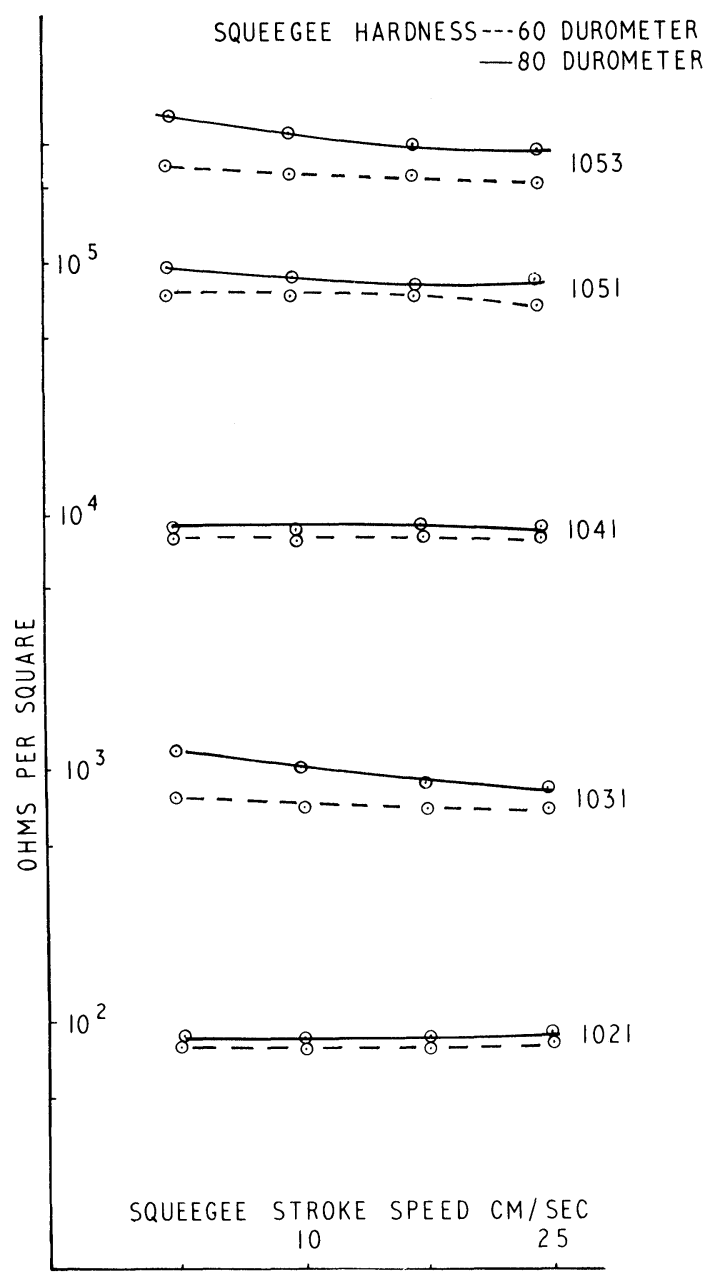

FIGURE 7 Sheet resistivity vs. squeegee speed.

It will by now be appreciated that any parameter which is capable of influencing the squeegee level during the printing stroke can cause a change in print thickness. In addition to stop position and squeegee traverse speed which have already been discussed, other parameters such as squeegee force, squeegee hardness, contact angle and screen tension all play a part.

\section{SQUEEGEE FORCE}

Squeegee force is usually applied by pneumatic pressure or by compressive springs. Since the applied force $F$ in printing mode A (see Figure 5(iii)) is spread uniformly across the substrate, the elastic deformation at the squeegee tip and therefore the level at which it operates is directly related to the substrate width.

For example, a $2.54 \mathrm{~cm} \times 1.27 \mathrm{~cm}$ substrate, orientated with its long side parallel to the squeegee, will require twice the squeegee force that needs to be applied with the short substrate side parallel to the squeegee to obtain the same print thickness, all other things being equal. In order to maintain printed thickness constant for differing substrate sizes, it is necessary to adjust the squeegee force. When doing so, it is prudent to remember to allow for inherent frictional forces.

Printing in a diagonal direction across square substrates is sometimes advocated, chiefly for reasons of symmetry associated with similarly shaped resistors lying both parallel and normal to an edge. In such cases the squeegee deformation under constant force increases steadily to a maximum half way across the substrate, and decreases whilst traversing the second half. This results in a corresponding print thickness variation causing resistors near the substrate centre to have lower resistivity than those situated in the leading and trailing corners. Elimination of such an embarassing situation is possible by rebating the substrate stage so that the substrate and stage surfaces are in one plane, thus maintaining a constant squeegee deformation throughout its stroke. In practice variations in substrate thickness and flatness detract from this ideal.

\section{SQUEEGEE HARDNESS AND CONTACT ANGLE}

Unfortunately it has not so far been possible to develop the preceding printing hypothesis into a formal mathematical discussion of the related effects of the squeegee hardness and its modified contact angle when travelling with a speed $V$ and under an applied force $F$.

However practical evidence shows that an increased squeegee force is necessary to compensate for squeegees of softer material. It is also known that reduced contact angles, as encountered with blade edges rounded with wear, require an increased squeegee force if print thickness is to be maintained.

\section{SCREEN TENSION}

As shown in Figure 5(ii), the downward force of the squeegee is opposed by components of the deflected screen tension. This opposing force is obviously 
dependent upon the degree of pre-tensioning used in the screen manufacture and is sensibly proportional to the amount of screen deflection which in printing is limited by the "snap off" distance. A minimum "snap off" distance of about $0.4 \mathrm{~mm}$ is necessary to accommodate variations in substrate thickness and flatness deficiencies if during printing the screen is to clear the wet ink columns. In practice, a more usual figure is $0.6 \mathrm{~mm}$. A suitably tensioned stainless steel screen mounted on an approximately $13 \mathrm{~cm}$ square frame and depressed by a $7.5 \mathrm{~cm}$ squeegee to this degree exerts an upward thrust of about $1 \mathrm{~kg}$ on the squeegee.

This thrust represents a significant portion of the squeegee force, and it makes good sense, not only to control closely the tension during screen manufacture, but to monitor that tension at intervals throughout the screen's active life. It is inevitable that screen tension will "slacken off" with continued use and rejection of a screen which has lost, say, 50 percent of its initial tension can help to maintain control of print thickness and prevent loss of printing definition also associated with this type of degradation.

\section{EFFECT OF SCREEN ORIENTATION}

With stainless steel screens it has been observed that the orientation of the mesh weave with respect to the printing stroke direction influences the print thickness, all other things being equal. It can be seen from Figure 8 that the cross section appearance of such a mesh is different in the two directions.

Referring to Figure 8(i), a squeegee blade printing in the direction into the paper experiences greater resistance to ink flow. The result is a raising of the squeegee level resulting in a print thickness about 10 percent greater than when printing into the paper in the case of Figure 8(ii). For good control, it is better

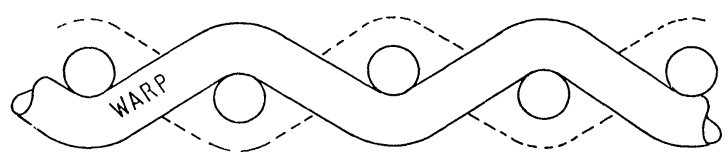

a.

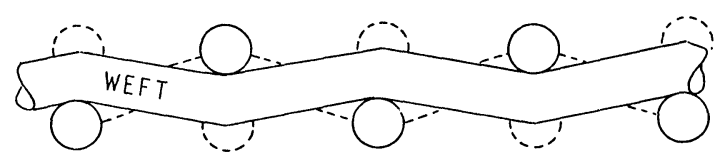

b.

FIGURE 8 Anisotropy of stainless steel mesh. to settle on one particular printing direction and persist with that.

\section{MISCELLANEOUS EFFECTS}

An additional source of print thickness variation can result from positional differences of the stencil with respect to the mesh grid. Consider the plan of part of a screen in Figure 9. A stencil defines three tracks $a, b$ and $c$. It can be seen that the prints resulting from tracks $a$ and $b$ will be identical, although the stencil - widths are clearly different.

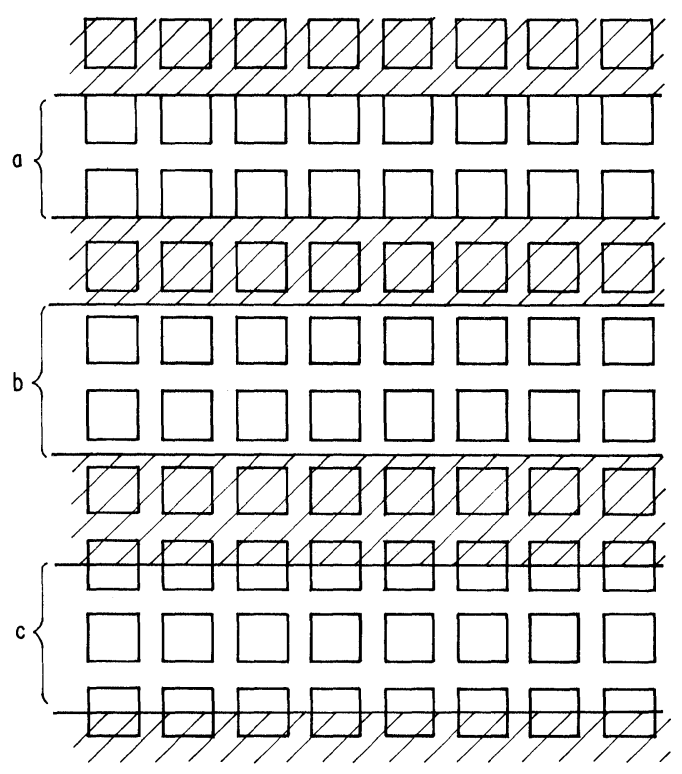

FIGURE 9 Positional effect of the stencil.

A third stencil track $c$ of equal width to $b$ will print the same volume of ink per unit stencil area as $b$ but the printed track width from $c$ will be wider, and in consequence its thickness less.

These examples demonstrate how the placement of the stencil with respect to the mesh grid can affect both printed track width and thickness. Variation of print thickness resulting from this kind of problem is predictable by the pillar theory and has been observed on samples of closely spaced narrow conducting tracks. Although negligible for tracks wider than about 0.050 in., this effect can result in a 10 percent thickness variation in worst case arrangements on tracks $0.010 \mathrm{in}$. wide or less. For this reason it is unlikely to be noticed on the majority of resistor prints. 
Nevertheless, designing the circuit pattern to a grid size which matches the printing screen mesh eliminates the problem. An even better answer is to set the mesh diagonally to the screen frame, whilst maintaining the stencil parallel. This system, although commonly used, is likely to be more wasteful of mesh material and would seem hardly worth the effort.

Even with the correct choice of screen material and with well tried printer parameters, when printing some circuit designs seemingly unaccountable resistance values are obtained.

Often it is one resistor value only in a complete network that does not conform and gives cause for concern. The cause of such an anomaly can sometimes be linked with a non-uniform pattern density of conducting track. Particularly in printing mode A a solitary resistor surrounded by densely packed conductor tracks will print thicker than one in a more open area.

This results from the squeegee level being higher in the more dense pattern area. Naturally the thicker the conducting layer, the greater this difference will be. In recorded cases resistors differing by 15 percent have resulted from this cause.

Closely associated with this problem is the tendency for small resistors to print thicker when orientated parallel rather than normal to the squeegee blade.

\section{CONCLUSIONS}

From the foregoing it is clear that a number of the problems encountered in controlling screened print thickness are topographical in nature, resulting from previously deposited layers and imperfect substrates. Other problems may be more basic and relate to the screen printing machine. For good print control it is essential that the squeegee blade traverses in a plane precisely parallel to the substrate stage. The parallelism of the screen is also important whilst the stroke speed, particularly in printing mode A, needs careful monitoring to ensure its constancy.

Finally, however good the printing control is, consistent batch to batch repeatability of resistance values cannot be expected unless the constancy of the subsequent firing process is assured. Some high valued resistive inks are very sensitive to the peak temperature experienced during the firing cycle. Resistance changes of 5 percent per degree centigrade difference in peak firing temperatures are not uncommon. Often such inks are ultra sensitive to print thickness too, so that achieving a batch to batch distribution of resistance values with a standard deviation of 10 percent is as much as can be expected.

On the other hand, usually at the lower end of the resistivity scale, inks tend to be more tolerant of processing conditions and corresponding standard deviations as low as 2 percent have been achieved.

\section{REFERENCES}

1. I. D. Salisbury "Variables in the thick-film screen printing process and their effect on register tolerances in large scale production". Electronic Components (April 1970).

2. B. M. Austin "Thick-film screen printing". Solid State Technology (June 1969).

3. R. W. Atkinson "Squeegee pressure and thick-film resistor fabrication". Solid State Technology (May 1971). 

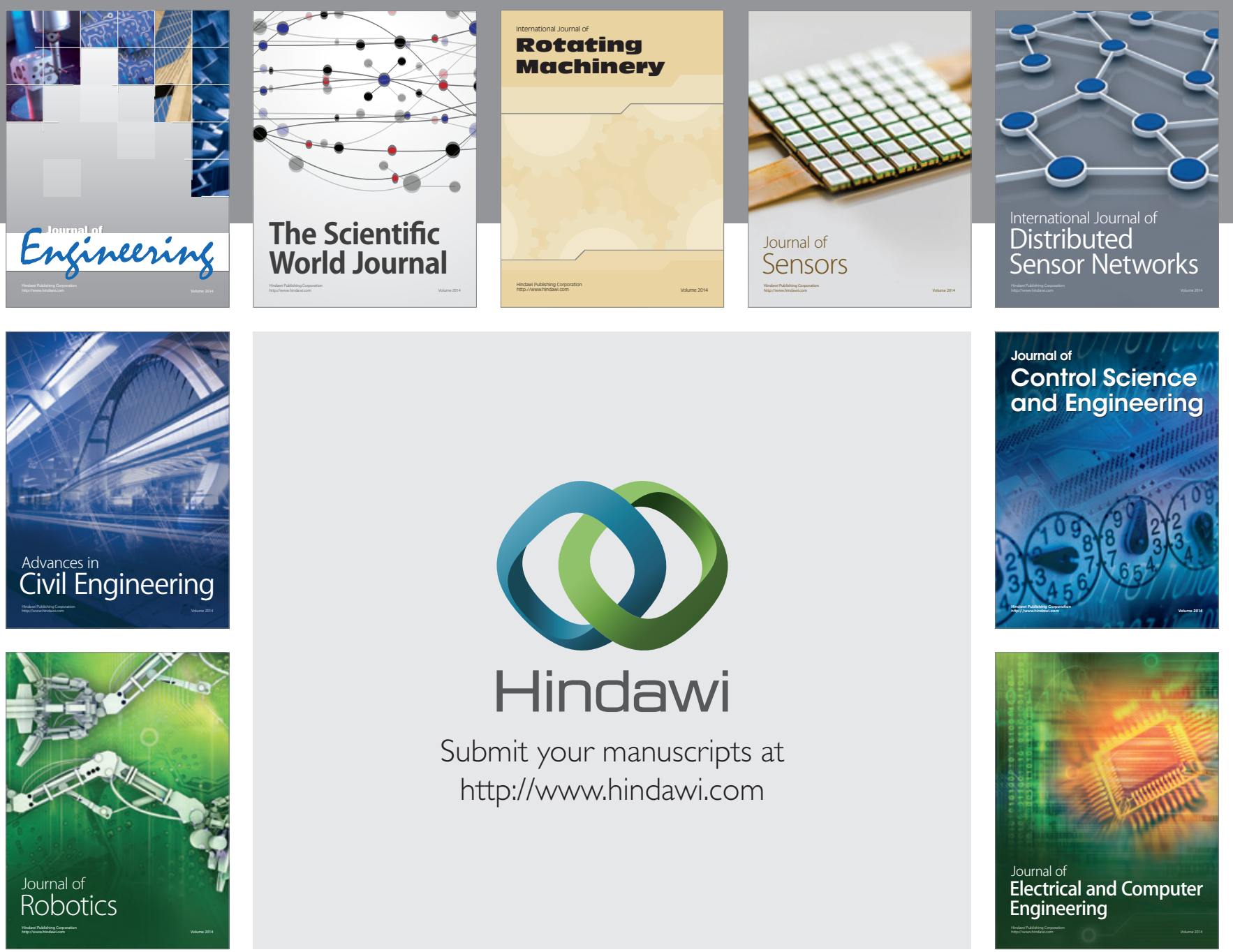

Submit your manuscripts at

http://www.hindawi.com
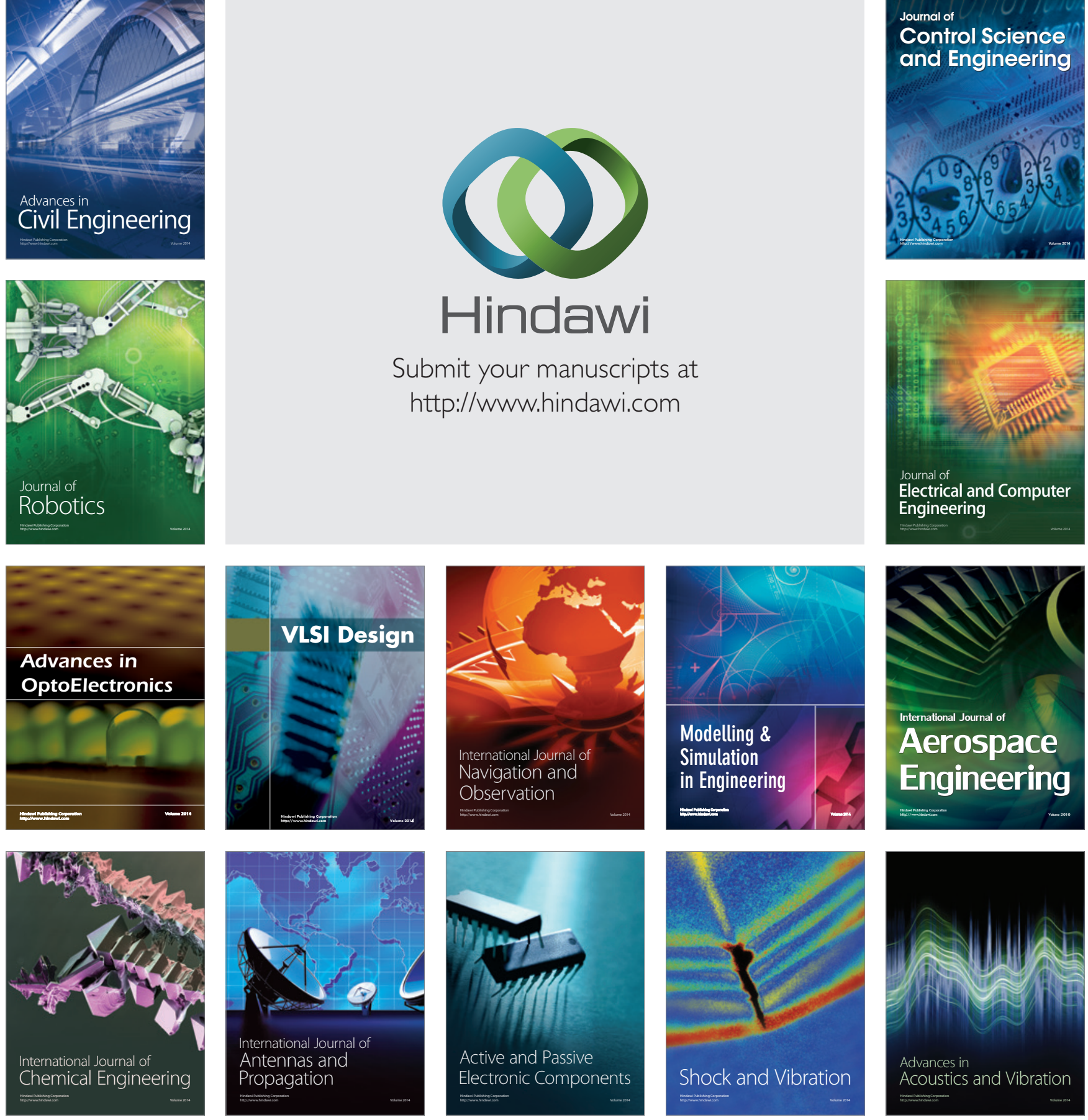